\title{
Kommentar zum Beitrag „Wunschkinder - Fördernde und hemmende Einflüsse medizinisch assistierter Reproduktion auf die Persönlichkeits- und Beziehungsentwicklung" von Frau Dr. Karin Lebersorger (neuropsychiatrie 2016;30:33-41)
}

\author{
Tewes Wischmann · Elmar Brähler · Heribert Kentenich · Yve Stöbel-Richter · Bernhard Strauß · Petra Thorn · \\ Julka Weblus
}

Online publiziert: 1 . September 2016

(C) Springer-Verlag Wien 2016

\section{Sehr geehrte Herausgeber!}

Ungewollt Kinderlose sind häufig mit einer Vielzahl von Belastungen konfrontiert: Neben dem unerfülltem Kinderwunsch und einer aufwändigen reproduktionsmedizinischen Behandlung sind sie nicht selten Vorurteilen des sozialen Umfelds und Stigmatisierungen ausgesetzt. Exemplarisch seien hier der Mythos der „psychischen Blockade“ als Ursache der Kinderlosigkeit genannt oder die Bezeichnung von Kindern nach assistierter Reproduktion als „Halbwesen“. Unverhältnismäßig pathologisierend sind nun auch viele Aussagen und Behauptungen im Beitrag

Priv.-Doz. Dr. sc.hum. Dipl.-Psych. T. Wischmann ( $\varangle)$ Institut für Medizinische Psychologie im Zentrum für Psychosoziale Medizin, Universitätsklinikum Heidelberg, Bergheimer Straße 20, 69115 Heidelberg, Deutschland tewes.wischmann@med.uni-heidelberg.de

\section{E. Brähler}

Abteilung für Medizinische Psychologie und Medizinische Soziologie, Universitätsklinikum Leipzig, Leipzig, Deutschland

H. Kentenich · J. Weblus

Fertility Center Berlin, Berlin, Deutschland

Y. Stöbel-Richter

Fakultät Management und Kulturwissenschaften,

Hochschule Zittau/Görlitz, Görlitz, Deutschland

\section{B. Strauß}

Institut für Psychosoziale Medizin und Psychotherapie, Universitätsklinikum, Friedrich-Schiller-Universität, Jena, Deutschland

P. Thorn

Praxis für Paar- und Familientherapie, Mörfelden,

Deutschland
„Wunschkinder - Fördernde und hemmende Einflüsse medizinisch assistierter Reproduktion auf die Persönlichkeits- und Beziehungsentwicklung“ von Frau Dr. Lebersorger: Die Inanspruchnahme der Reproduktionsmedizin gehe mit Spaltung, Verleugnung, Verdrängung, Überflutung von narzisstischer Kränkung, Schuldgefühlen und dem Wunsch des „kontrollierten Gebärens“ einher, so dass „medizinisch assistierte Reproduktion per se eine Risikokonstellation für die emotionale Entwicklung eines Kindes“ darstelle. Ein Blick in die Leitlinie „Psychosomatisch orientierte Diagnostik und Therapie bei Fertilitätsstörungen“ (s. www.leitlinien.net) wäre ausreichend gewesen, um sich an den aktuellen Ergebnissen evidenzbasierter Medizin zur Thematik zu orientieren. Darin wird aufgezeigt, dass eine überwiegend psychopathologieorientierte Betrachtung der Paare auf Grundlage wissenschaftlicher Befunde keineswegs gerechtfertigt ist, und dass auch die Eltern-Kind-Beziehung bzw. die Entwicklung von Einlingen nach ART (im Vergleich zu spontan konzipierten Einlingen) weitgehend unauffällig ist. So ist der Beitrag von Fr. Dr. Lebersorger unseres Erachtens leider nicht geeignet, ungewollt kinderlosen Paaren eine hilfreiche Orientierung in dieser oft als sehr schwierig erlebten Lebensphase an die Hand zu geben und auch psychosoziale und medizinische Professionelle in diesem Feld werden ihn im Jahr 2016 kaum als fachlich bereichernd wahrnehmen können.

Interessenkonflikt T. Wischmann, E. Brähler, H. Kentenich, Y. Stöbel-Richter, B. Strauß, P. Thorn und J. Weblus sind Mitglieder der AWMF-Leitliniengruppe „Psychosomatisch orientierte Diagnostik und Therapie bei Fertilitätsstörungen“. 